\title{
Analysis of Phenolic Compounds in Commercial Cannabis sativa L. Inflorescences Using UHPLC-Q-Orbitrap HRMS
}

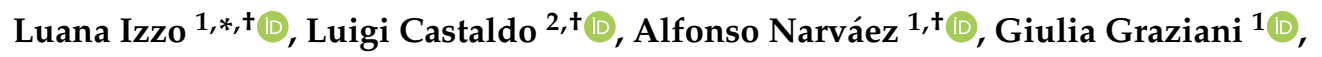 \\ Anna Gaspari ${ }^{1}{ }^{1}$, Yelko Rodríguez-Carrasco ${ }^{3}\left(\mathbb{D}\right.$ and Alberto Ritieni ${ }^{1}$ \\ 1 Department of Pharmacy, Faculty of Pharmacy, University of Naples “Federico II," Via Domenico \\ Montesano 49, 80131 Naples, Italy; alfonso.narvaezsimon@unina.it (A.N.); giulia.graziani@unina.it (G.G.); \\ annagaspari@virgilio.it (A.G.); alberto.ritieni@unina.it (A.R.) \\ 2 Department of Clinical Medicine and Surgery, University of Naples "Federico II", Via S. Pansini 5, \\ 80131 Naples, Italy; luigi.castaldo2@unina.it \\ 3 Laboratory of Food Chemistry and Toxicology, Faculty of Pharmacy, University of Valencia, Av. Vicent \\ Andrés Estellés s/n, 46100 Burjassot, Spain; yelko.rodriguez@uv.es \\ * Correspondence: luana.izzo@unina.it; Tel.: +39-081-678116 \\ + These authors contributed equally to this work.
}

Academic Editor: Thomas Efferth

Received: 30 December 2019; Accepted: 25 January 2020; Published: 31 January 2020

\begin{abstract}
Industrial hemp (Cannabis sativa L. Family Cannabaceae) contains a vast number of bioactive relevant compounds, namely polyphenols including flavonoids, phenolic acids, phenol amides, and lignanamides, well known for their therapeutic properties. Nowadays, many polyphenols-containing products made of herbal extracts are marketed, claiming to exert health-promoting effects. In this context, industrial hemp inflorescence may represent an innovative source of bioactive compounds to be used in nutraceutical formulations. The aim of this work was to provide a comprehensive analysis of the polyphenolic fraction contained in polar extracts of four different commercial cultivars (Kompoti, Tiborszallasi, Antal, and Carmagnola Cs) of hemp inflorescences through spectrophotometric (TPC, DPPH tests) and spectrometry measurement (UHPLC-Q-Orbitrap HRMS). Results highlighted a high content of cannflavin A and B in inflorescence analyzed samples, which appear to be cannabis-specific, with a mean value of 61.8 and $84.5 \mathrm{mg} / \mathrm{kg}$, meaning a ten-to-hundred times increase compared to other parts of the plant. Among flavonols, quercetin-3-glucoside reached up to $285.9 \mathrm{mg} / \mathrm{kg}$ in the Carmagnola CS cultivar. Catechin and epicatechin were the most representative flavanols, with a mean concentration of 53.3 and $66.2 \mathrm{mg} / \mathrm{kg}$, respectively, for all cultivars. Total polyphenolic content in inflorescence samples was quantified in the range of 10.51 to $52.58 \mathrm{mg}$ GAE/g and free radical-scavenging included in the range from 27.5 to $77.6 \mathrm{mmol}$ trolox $/ \mathrm{kg}$. Therefore, $C$. sativa inflorescence could be considered as a potential novel source of polyphenols intended for nutraceutical formulations.
\end{abstract}

Keywords: Cannabis sativa L.; polyphenols; UHPLC-Q-Orbitrap HRMS

\section{Introduction}

Cannabis sativa is an annual herbaceous plant of the Cannabaceae family native to Central Asia, but with a wide distribution over different geographical areas facilitated by climate adaptation. This plant has long been cultivated due to its large variety of applications, from textile uses to food and feed [1].

Industrial hemp, characterized by a low content of psychoactive cannabinoids, contains bioactive compounds that are known to have a wide range of important biological properties [2]. Polyphenols represent one of the most relevant compounds found in C. sativa, such as prenylated flavonoids, 
phenol amides, and lignanamides, which are specific metabolites of this plant. They are known to play multifunctional roles in the defense mechanisms of the plant, especially through their activity as antioxidants, preventing the generation of reactive oxygen species (ROS) [3-6]. In humans, polyphenols can display health-promoting effects based on the modulation of several enzymes, such us lipoxygenase and cytochrome P450 system, showing cardio or chemoprotective activity, among others [5,7].

For this reason, polyphenols-containing products have been marketed over the last years as food supplements and nutraceuticals, and, currently, a great variety of supplements claiming to enhance specific physiological functions are commercially available. Nutraceuticals consist of naturally-occurring active substances, which are concentrated and administered in the suitable pharmaceutical form to properly develop its pharmacological effect. Furthermore, when compared to traditional drugs, nutraceuticals appear to be generally safer, with higher bioavailability and fewer side effects [8]. The manufacturing of nutraceuticals requires isolated ingredients that have to be extracted and purified for latter uses. Since certain polyphenols naturally occur inside insoluble structures, such as vacuoles, obtention of pure compounds can become a complex process [9]. In addition, several studies reported a decrease in the bioavailability and bioaccessibility of pure polyphenols in comparison with the administration of plant extracts rich in polyphenols, which may be due to the existence of other active compounds which can establish synergistic functions with them [10-12]. Because of this, food supplements could be a valuable resource to consume polyphenols-containing products. They consist of extracts from herbals and botanicals than can be delivered as the same pharmaceutical forms as nutraceuticals. Some of the most prevalent plants used as a source of polyphenols are tea, coffee, apple, basil, and turmeric, among others, each one intended for specific polyphenols [13-15].

Regarding C. sativa, recent studies have reported the high antioxidant potential of the plant, also characterizing the major polyphenols, $\mathrm{N}$-trans-caffeoyltyramine, and cannabisin $\mathrm{A}, \mathrm{B}$ and $C$, and concluding that $C$. sativa would be a suitable source of polyphenols for nutraceutical or supplementation purposes [3,4,16-18]. Nevertheless, the most studied organs of the plant are seeds, leaves, and sprouts, whereas there is still scarce literature regarding polyphenols in inflorescences. The polyphenolic profile of $C$. sativa is variable among the different parts of the plant, and since flowers represent an important reproductive organ, high levels of colored polyphenols are expected [19].

Analysis of polyphenols in C. sativa samples have been previously performed using Fourier transform infrared (FTIR) spectroscopy with attenuated total reflectance (ATR) [4], mass spectrometry (MS) coupled to both high-performance liquid chromatography (HPLC), and gas chromatography (GC) [18]. High-resolution mass spectrometers, such as Orbitrap, have also been used coupled to ultra-high performance liquid chromatography (UHPLC) for the determination of polyphenols in vegetal matrices intended for nutraceutical purposes, including green tea and coffee [17,20-23]. This methodology offers higher sensitivity and specificity, allowing a precise quantification based on exact mass measurement. Therefore, the aim of this study was to (i) evaluate the antioxidant activity and total polyphenol content in different chemotypes of commercial C. sativa inflorescences using in vitro assays and (ii) to establish the polyphenolic profile through ultra-high-performance liquid chromatography coupled to a high-resolution Orbitrap mass spectrometry, to promote the use of this innovative source of bioactive compounds to be used in nutraceutical gformulations or for their health-promoting properties.

\section{Results and Discussion}

\subsection{Identification of Polyphenols Compounds in C. sativa Inflorescences though UHPLC-Q-Orbitrap HRMS}

Identification of individual phenolic acids and flavonoids was conducted through UHPLC-Q-Orbitrap HRMS. By a combination of MS and MS/MS spectra, a total of 22 different polyphenolic compounds were identified from different samples of $C$. sativa inflorescences (Figures $\mathrm{S} 1$ and $\mathrm{S} 2$ ). Table 1 shows all mass parameters including adduct ion, theoretical and measured mass $(m / z)$, accuracy and sensitivity. 
Table 1. Chromatographic and spectrometric optimized parameters including retention time, adduct ion, theoretical and measured mass $(\mathrm{m} / \mathrm{z})$, accuracy and sensibility for the investigated analytes $(n=22)$.

\begin{tabular}{|c|c|c|c|c|c|c|c|c|}
\hline Compound & $\begin{array}{l}\text { ention Time } \\
(\min )\end{array}$ & $\begin{array}{l}\text { Chemical } \\
\text { Formula }\end{array}$ & Adduct Ion & $\begin{array}{l}\text { Theoretical } \\
\text { Mass }(\mathrm{m} / \mathrm{z})\end{array}$ & $\begin{array}{c}\text { Measured Mass } \\
(\mathrm{m} / \mathrm{z})\end{array}$ & $\begin{array}{l}\text { Accuracy } \\
(\Delta \mathrm{mg} / \mathrm{kg})\end{array}$ & LOD (mg/kg) & LOQ (mg/kg) \\
\hline Catechin & 7.65 & $\mathrm{C}_{15} \mathrm{H}_{14} \mathrm{O}_{6}$ & {$[\mathrm{M}-\mathrm{H}]^{-}$} & 289.07176 & 289.07224 & 1.6605 & 0.0015 & 0.0046 \\
\hline $\begin{array}{l}\text { Chlorogenic } \\
\text { acid }\end{array}$ & 8.13 & $\mathrm{C}_{16} \mathrm{H}_{18} \mathrm{O}_{9}$ & {$[\mathrm{M}-\mathrm{H}]^{-}$} & 353.08780 & 353.08798 & 0.5098 & 0.0012 & 0.0036 \\
\hline Caffeic acid & 8.24 & $\mathrm{C}_{9} \mathrm{H}_{8} \mathrm{O}_{4}$ & {$[\mathrm{M}-\mathrm{H}]^{-}$} & 179.03498 & 179.03455 & -2.4018 & 0.0007 & 0.0020 \\
\hline Epicatechin & 8.51 & $\mathrm{C}_{15} \mathrm{H}_{14} \mathrm{O}_{6}$ & {$[\mathrm{M}-\mathrm{H}]^{-}$} & 289.07176 & 289.07196 & 0.6919 & 0.0014 & 0.0043 \\
\hline Luteolin-7-O-glucoside & 9.23 & $\mathrm{C}_{21} \mathrm{H}_{20} \mathrm{O}_{11}$ & {$[\mathrm{M}-\mathrm{H}]^{-}$} & 447.09328 & 447.09366 & 0.8499 & 0.0008 & 0.0025 \\
\hline$p$-Coumaric acid & 9.31 & $\mathrm{C}_{9} \mathrm{H}_{8} \mathrm{O}_{3}$ & {$[\mathrm{M}-\mathrm{H}]^{-}$} & 163.04001 & 163.03937 & -3.9254 & 0.0006 & 0.0018 \\
\hline $\begin{array}{l}\text { Caffeoyl } \\
\text { tyramine }\end{array}$ & 9.46 & $\mathrm{C}_{17} \mathrm{H}_{17} \mathrm{NO}_{4}$ & {$[\mathrm{M}-\mathrm{H}]^{-}$} & 298.10848 & 298.10910 & 2.0798 & - & - \\
\hline Rutin & 9.79 & $\mathrm{C}_{27} \mathrm{H}_{30} \mathrm{O}_{16}$ & {$[\mathrm{M}-\mathrm{H}]^{-}$} & 609.14611 & 609.14624 & 0.2134 & 0.0012 & 0.0035 \\
\hline Ferulic acid & 9.88 & $\mathrm{C}_{10} \mathrm{H}_{1} \mathrm{O}_{4}$ & {$[\mathrm{M}-\mathrm{H}]^{-}$} & 193.05063 & 193.05016 & -2.4346 & 0.0018 & 0.0054 \\
\hline Quercetin-3-glucoside & 9.93 & $\mathrm{C}_{20} \mathrm{H}_{20} \mathrm{O}_{12}$ & {$[\mathrm{M}-\mathrm{H}]^{-}$} & 463.08820 & 463.08862 & 0.9070 & 0.0017 & 0.0052 \\
\hline Kaempferol-3-O-glucosid & dळ0.36 & $\mathrm{C}_{21} \mathrm{H}_{20} \mathrm{O}_{11}$ & {$[\mathrm{M}-\mathrm{H}]^{-}$} & 447.09323 & 447.09360 & 0.8276 & 0.0008 & 0.0025 \\
\hline Apigenin-7-glucoside & 10.36 & $\mathrm{C}_{21} \mathrm{H}_{20} \mathrm{O}_{10}$ & {$[\mathrm{M}-\mathrm{H}]^{-}$} & 431.09837 & 431.09836 & -0.0232 & 0.0004 & 0.0013 \\
\hline Cannabisin A & 10.54 & $\mathrm{C}_{34} \mathrm{H}_{30} \mathrm{~N}_{2} \mathrm{O}_{8}$ & {$[\mathrm{M}-\mathrm{H}]^{-}$} & 593.19294 & 593.19281 & -0.2192 & - & - \\
\hline Quercetin & 11.00 & $\mathrm{C}_{15} \mathrm{H}_{10} \mathrm{O}_{7}$ & {$[\mathrm{M}-\mathrm{H}]^{-}$} & 301.03538 & 301.03508 & -0.9966 & 0.0021 & 0.0064 \\
\hline Luteolin & 11.25 & $\mathrm{C}_{15} \mathrm{H}_{10} \mathrm{O}_{6}$ & {$[\mathrm{M}-\mathrm{H}]^{-}$} & 285.04046 & 285.04050 & 0.1403 & 0.0004 & 0.0012 \\
\hline Cannabisin B & 11.41 & $\mathrm{C}_{34} \mathrm{H}_{32} \mathrm{~N}_{2} \mathrm{O}_{8}$ & {$[\mathrm{M}-\mathrm{H}]^{-}$} & 595.20859 & 595.20709 & -2.5201 & - & - \\
\hline Kaempferol & 11.60 & $\mathrm{C}_{15} \mathrm{H}_{10} \mathrm{O}_{6}$ & {$[\mathrm{M}-\mathrm{H}]^{-}$} & 285.04046 & 285.04086 & 1.4033 & 0.0005 & 0.0014 \\
\hline Naringenin & 11.78 & $\mathrm{C}_{15} \mathrm{H}_{12} \mathrm{O}_{5}$ & {$[\mathrm{M}-\mathrm{H}]^{-}$} & 271.06120 & 271.06146 & 0.9592 & 0.0005 & 0.0015 \\
\hline Apigenin & 11.85 & $\mathrm{C}_{15} \mathrm{H}_{10} \mathrm{O}_{5}$ & {$[\mathrm{M}-\mathrm{H}]^{-}$} & 269.04555 & 269.04572 & 0.6319 & 0.0004 & 0.0011 \\
\hline Cannabisin $\mathrm{C}$ & 12.34 & $\mathrm{C}_{35} \mathrm{H}_{34} \mathrm{~N}_{2} \mathrm{O}_{8}$ & {$[\mathrm{M}-\mathrm{H}]^{-}$} & 609.22424 & 609.22485 & 1.0013 & - & - \\
\hline Cannflavin B & 13.77 & $\mathrm{C}_{21} \mathrm{H}_{20} \mathrm{O}_{6}$ & {$[\mathrm{M}-\mathrm{H}]^{-}$} & 367.11871 & 367.11871 & 0.000 & - & - \\
\hline Cannflavin A & 14.84 & $\mathrm{C}_{26} \mathrm{H}_{28} \mathrm{O}_{6}$ & {$[\mathrm{M}-\mathrm{H}]^{-}$} & 435.18131 & 435.18143 & 0.2757 & - & - \\
\hline
\end{tabular}


Experiments were achieved in $\mathrm{ESI}^{-}$mode. All of the studied analytes exhibited better fragmentation patterns producing the quasi-molecular ion $[\mathrm{M}-\mathrm{H}]^{-}$. After full scan analysis, the accurate mass of the characteristic ions (precursor ions) was included in an inclusion list.

Full-scan HRMS data acquisition captures all sample data, enabling the identification of untargeted compounds and retrospective data analysis without the need to re-run samples. The confirmation of the structural characterization of untargeted analytes was based on the accurate mass measurement, elemental composition assignment, and MS/MS spectrum interpretation (Figure S3).

Optimal separation of all the investigated analytes was carried out in a total run time of $20 \mathrm{~min}$. The identification of structural isomers: catechin and epicatechin $(\mathrm{m} / \mathrm{z} 289.07176)$; luteolin and kaempferol $(\mathrm{m} / \mathrm{z} 285.04046)$, was achieved by comparing the retention times of the peaks with those of standards (Figure S2).

Sensitivity was evaluated by the limit of detection (LOD) and limit of quantification (LOQ). The LOD was defined as the minimum concentration, where the molecular ion could be identified with a mass error below $5 \mathrm{ppm}$, and the LOQ was set as the lowest concentration of the analyte that produced a chromatographic peak with a precision and accuracy $<20 \%$.

Quantitative determination of target analytes $(n=16)$ was performed using calibration curves at eight concentration levels. Each calibration curve was prepared in triplicate. We obtained regression coefficients $>0.990$. Quantification of compounds $(n=6)$ that had no standard to generate a curve was based on a representative standard of the same group.

\subsection{Quantification of Phenolic Acids and Flavonoids in C. sativa Inflorescences}

\subsubsection{Phenolic Acids}

The predominant lignanamides (cannabisin A, B, and C) and phenolic amide ( $N$-trans-caffeoyltyramine) found in hemp were evaluated in the assayed samples. Lignanamides and phenolic amides belong to the lignan class of compounds, and the basic unit consists of tyramine condensed with CoA-esters of $p$-coumaric, caffeic and coniferic acid, as suggested by Flores-Sanchez [24]. Table 2 shows the results here obtained expressed as the average content and concentration range of the phenolic acids and flavonoids detected in different hemp varieties. In the here analyzed samples, lignanamides represented from $0.02 \%$ to $0.47 \%$ of total polyphenols in a concentration range between 0.10 and $2.2 \mathrm{mg} / \mathrm{kg}$. Cannabisin A was found as the most commonly detected lignanamide ranging from 0.01 (Tiborszallasi) up to $2.86 \mathrm{mg} / \mathrm{kg}$ (Kompolti), with a mean value of $1.0 \mathrm{mg} / \mathrm{kg}$ for all cultivars. Cannabisin B was found at levels three times lower with respect to Cannabisin A, ranging from 0.4 to $0.5 \mathrm{mg} / \mathrm{kg}$. In addition, when cannabisin A was found at very low concentrations, cannabisin B was not detected. Cannabisin $C$ showed to be the less relevant lignanamide, quantified between 0.003 and $0.38 \mathrm{mg} / \mathrm{kg}$. Concerning the occurrence of lignanamides in C. sativa seed, available studies reported the highest concentration up to thousands milligram per kilogram [25]. As far as phenol amides were concerned, $\mathrm{N}$-trans-caffeoyltyramine was quantified at a concentration range from 0.1 (Kompolti) to $76.2 \mathrm{mg} / \mathrm{kg}$ (Carmagnola Cs), with a mean value of $23.7 \mathrm{mg} / \mathrm{kg}$ for all cultivars. These levels are in line with the data reported in hemp seed [25]. Lignanamides and phenolic amides are known to have a wide range of important biological properties, including antioxidant, anti-inflammatory, and antihyperlipidemic activities [26-30]. Apart from those, some important hydroxycinnamic acids (chlorogenic acid, caffeic acid, $p$-coumaric acid, and ferulic acid) were evaluated in the here analyzed inflorescences samples. This important class of phenolic acids represented from $18.6 \%$ to $29.7 \%$ of total polyphenols found in samples. Among the hydroxycinnamic acids, $p$-coumaric acid was quantified at concentrations significantly greater $(p<0.05)$ than the other related compounds in all hemp cultivars analyzed except in Kompolti samples. Moreover, the most common hydroxycinnamic acids found in Kompolti cultivar was ferulic acid at an average content of $19.7 \mathrm{mg} / \mathrm{kg}$ (range from 3.0 to $35.6 \mathrm{mg} / \mathrm{kg}$ ). Caffeic acid was detected in the lowest amount for all the analyzed cultivars. Carmagnola Cs hemp variety showed the highest concentration of hydroxycinnamic acids compared with other varieties 
at an average content of $85.4 \mathrm{mg} / \mathrm{kg}$. On the other hand, the observed concentration variability of phenolic acids may be a result of the influence of many biotic and abiotic factors that play an important role in the biosynthetic process of the studied compounds [31].

Table 2. Polyphenols content in the analyzed Cannabis sativa samples $(n=22)$. Results are shown based on the different cultivar C. sativa inflorescences.

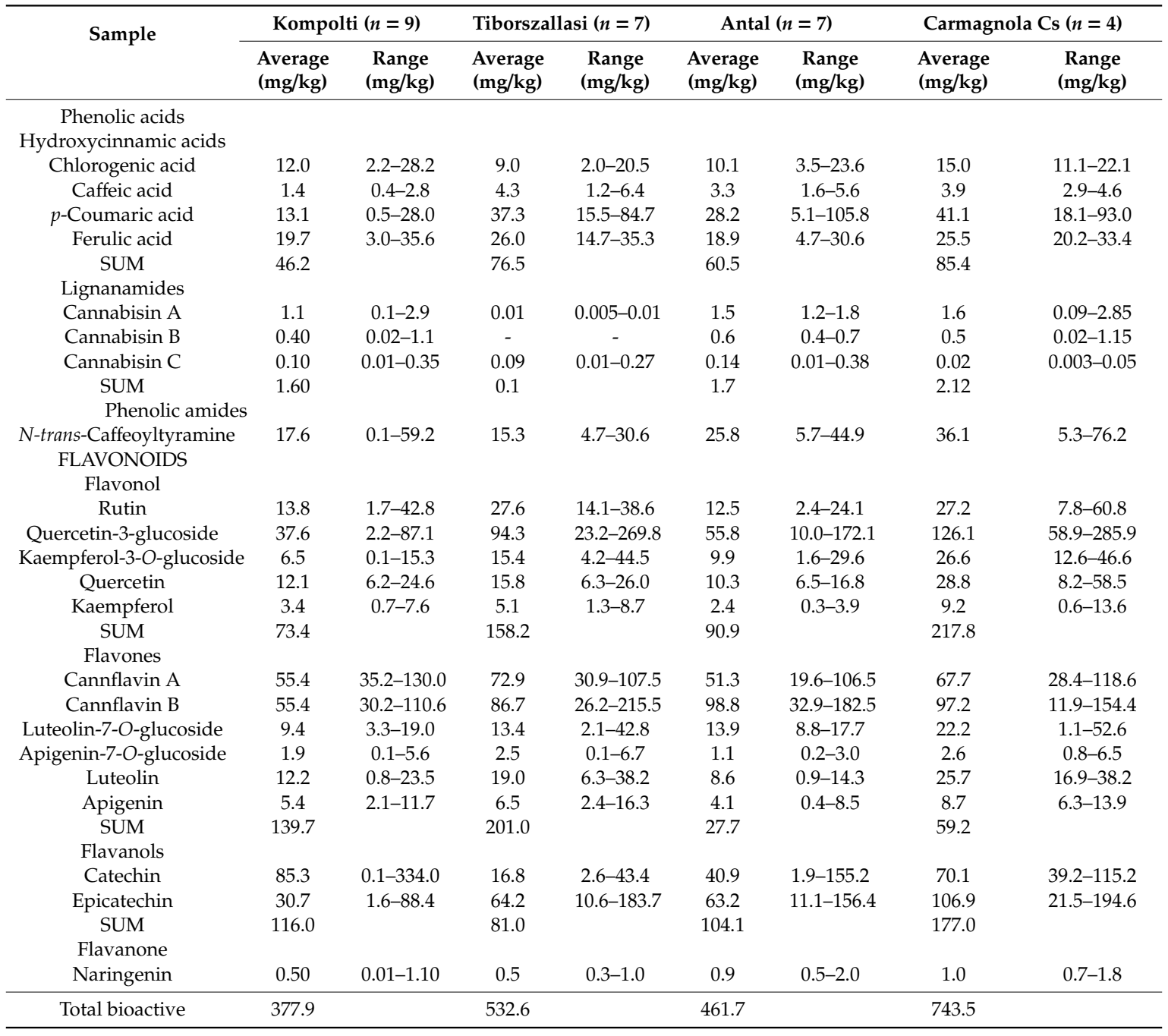

\subsubsection{Flavonoids}

Flavonoids are plant-derived phytochemicals that accounted for over $80 \%$ of the phenolic components in the assayed samples. Flavonoids, namely flavones, flavanones, flavonols, and flavanols, including their main aglycones, glycosides, and methylated derivatives, have been quantified in the assayed samples as shown in Table 2. Flavones represented the highest proportion of flavonoids found in analyzed samples, ranging from 30.1\% (Carmagnola Cs) to 35.4\% (Kompolti) of total polyphenols, cannflavin A and cannflavin B being the most commonly detected flavones, with a mean value of 61.8 and $84.5 \mathrm{mg} / \mathrm{kg}$, respectively. These levels found in inflorescences showed a ten-to-hundred-fold increase when compared to leaves samples previously analyzed by Pollastro et al., [5] who reported cannflavin A and B at 6 and $0.8 \mathrm{mg} / \mathrm{kg}$, respectively. Cannflavins A and B, methylated isoprenoid flavones, appear to be Cannabis specific and are known to exert a potent anti-inflammatory activity [32]. In this context, Werz et al. [33] reported that cannflavin A and B were able to inhibit the production of pro-inflammatory prostanoids and leukotrienes in in vitro assays. Moreover, Barrett et al. concluded that cannflavins A and B promoted the inhibition of PGE2 in human cells up to thirty times in relation to 
aspirin [34,35]. In addition, cannflavin A showed a neuroprotective effect against amyloid $\beta$-mediated neurotoxicity in PC12 cells [36]. As shown in Table 2, among flavonols, quercetin-3-glucoside was quantified at concentrations significantly greater $(p<0.05)$ than the other related compounds, ranging from 2.2 (Kompolti) to $285.9 \mathrm{mg} / \mathrm{kg}$ (Carmagnola Cs) with a mean value of $78.4 \mathrm{mg} / \mathrm{kg}$. On the other hand, flavanols mainly represented by catechin and epicatechin were detected at mean values of 53.3 and $66.2 \mathrm{mg} / \mathrm{kg}$ for all cultivars, respectively. Regarding epicatechin, Carmagnola Cs cultivar showed a two-fold increase in the mentioned-flavonol compared with other cultivars assayed. The minor flavonoids compound detected was naringenin.

Overall, the data clearly indicate that Carmagnola Cs cultivar showed the highest concentrations of the investigated polyphenols $(743.5 \mathrm{mg} / \mathrm{kg})$ compared to the other cultivars.

Industrial hemp inflorescence may represent an innovative source of bioactive compounds, such as a high content of cannflavin A and B, which appear to be cannabis-specific to be used in nutraceutical formulations.

\subsection{Total Phenolic Contents and Antioxidant Activity of C. sativa Extracts}

\subsubsection{Total Phenolic Contents of $C$. sativa Extracts}

The amounts of total phenolic contents of $C$. sativa extracts were examined. The results are summarized in Table 3. Total phenolics, flavonoids, and phenolic acids ranged from 10.510 to $48.875 \mathrm{mg}$ GAE/g for the different cultivars of C. sativa. Among the studied cultivars, the highest total phenols content resulted from Carmagnola Cs with the content of $41.517 \mathrm{mg} \mathrm{GAE} / \mathrm{g}$ and, the lower amount emerges for the Kompolti variety (10.510 mg GAE/g). Total polyphenol content quantified in analyzed samples was similar to those recently reported by Vonapartis et al., [37] in hemp seeds samples $(n=10)$ in a concentration range from 13.68 to $51.60 \mathrm{mg} \mathrm{GAE} / \mathrm{g}$.

Table 3. Total polyphenol compounds expressed as mg GAE/g and antioxidant activity expressed as mmol trolox $/ \mathrm{kg}$ of C. sativa extracts.

\begin{tabular}{ccccc}
\hline \multirow{2}{*}{ Cultivar } & \multicolumn{2}{c}{ TPC } & \multicolumn{2}{c}{ DPPH } \\
\cline { 2 - 5 } & $\begin{array}{c}\text { Average } \\
\text { (mg GAE/g) }\end{array}$ & Range (mg GAE/g) & $\begin{array}{c}\text { Average } \\
\text { (mmol trolox/kg) }\end{array}$ & $\begin{array}{c}\text { Range } \\
\text { (mmol trolox/kg) }\end{array}$ \\
\hline Kompolti $(n=9)$ & $26.2 \pm 0.5$ & $10.5-47.2$ & $46.7 \pm 0.7$ & $36.6-55.0$ \\
Tiborszallasi $(n=7)$ & $29.9 \pm 0.4$ & $21.9-42.9$ & $61.3 \pm 0.9$ & $50.8-72.7$ \\
Antal $(n=7)$ & $30.7 \pm 0.5$ & $17.0-48.9$ & $45.9 \pm 0.4$ & $27.5-67.5$ \\
Carmagnola Cs $(n=4)$ & $33.2 \pm 0.5$ & $26.3-41.5$ & $63.6 \pm 0.9$ & $59.1-77.6$ \\
\hline
\end{tabular}

${ }^{*}$ GAE: gallic acid equivalents; TPC: total phenolic content; DPPH: free-radical scavenging; trolox: equivalent antioxidant capacity (TEAC).

C. sativa is a remarkable plant widely investigated by several researchers, given its rich fount of valuable natural components. Apart from cannabinoids production, the $C$. sativa plant is also able to synthesize non-cannabinoids second metabolites possessing benefic effects for human health $[1,38]$. A summary of the available surveys of total polyphenols contents of the different parts of $C$. sativa is shown in Table 4. In spite of several experiments were done on this plant [3,4,17,38-43], the study of inflorescence remains low, making it difficult the comparison of results. Recently, Ferrante et al. [25] investigated the total phenolic content in the water fraction extracted from aerial flowers of Carmagnola Cs cultivar, reporting a lower value compared to the results here obtained.

With respect to other parts of the same plant, the given TPC in C. sativa inflorescences was significantly $(p<0.05)$ higher than the reported contents, resulting in the highest potential fount of phenols.

The correlation between TPC and UHPLC-Q-Orbitrap HRMS findings was evaluated through Pearson's correlation coefficient (PCC). The results showed a strong positive correlation ( $P C C=0.892)$. 
Scientific evidence suggests that using some combination of assays is the best approach to properly characterize the phenolic composition [44].

Table 4. Recent surveys reporting the total phenolic content (mg GAE/g) in different parts of C. sativa samples.

\begin{tabular}{ccc}
\hline Part of Plant & TPC $(\mathbf{m g}$ GAE $/ \mathbf{g})$ & References \\
\hline Leaves & $0.09-0.56$ & {$[3]$} \\
Seeds & $0.77-51.6$ & {$[4,17,37,39]$} \\
Oil & $0.02-2.67$ & {$[4,39,40]$} \\
Flour & $0.74-1.71$ & {$[4,39,43]$} \\
Sprouts & 6.16 & {$[17]$} \\
Aerial parts & $5.85-17.05$ & {$[41]$} \\
Flowers & $4.7-8.1$ & {$[42]$} \\
Inflorescences & $10.51-52.58$ & Current work \\
\hline
\end{tabular}

\subsubsection{Antioxidant Activity of $C$. sativa Extracts}

The results of antioxidant activity evaluated through DPPH free radical-scavenging activity are tabulated in Table 3 and expressed as mmol trolox $/ \mathrm{kg}$. A calibration curve of inhibition, built with trolox@, was employed as a positive control of the assay. Antioxidant activity was included in the range of 27.532 to $77.578 \mathrm{mmol}$ trolox $/ \mathrm{kg}$ (average $54.401 \mathrm{mmol}$ trolox $/ \mathrm{kg}$ ) for the different cultivars of C. sativa. The highest antioxidant capability was shown, in this case, in Carmagnola Cs $(p<0.05)$ with a content of $77.578 \mathrm{mmol}$ trolox $/ \mathrm{kg}$ and the lower amount in the Antal variety $(27.532 \mathrm{mmol}$ trolox $/ \mathrm{kg})$.

Antioxidant capacity is largely used as a parameter to characterize bioactive components from foods or medicinal plants. The assessment of polyphenols contents in inflorescences of C. sativa extract is useful to define their potential antioxidant value and their free radical scavenging capacity. Increasing evidence reports that antioxidants may protect cell constituents against oxidative damage and shrink the risk of various diseases connected to oxidative stress [45].

The synergy effect or interaction of phenolic compounds in food products contribute, for the most part, to the overall antioxidant capacity, despite bioactivity decoupled from phenolic compounds being reported in the literature [46,47].

Several researchers have studied the bioactivity of various parts of C. sativa $[3,4,17,39-43]$, and, on the whole, the extracts were found to be rich sources of bioactive compounds. As regards antioxidant capability, Smeriglio et al. [40] reported contents of $146.7 \mathrm{mmol}$ trolox/100 g in seed oil from Finola cultivar and lower amounts for the lipophilic fraction $(0.125 \mathrm{mmol}$ trolox $/ 100 \mathrm{~g})$ and hydrophilic fractions (0.038 mmol trolox/100 g). Instead, Mikulek et al., [43] have reported an amount of $158 \mathrm{mmol}$ trolox $/ 100 \mathrm{~kg}$ in flour.

Currently, scarce data on the antioxidant activity of $C$. sativa inflorescence are available in the literature. Compared to other findings that reported the antioxidant activity of the different parts of C. sativa, the inflorescence showed the highest properties.

\section{Materials and Methods}

\subsection{Reagents and Materials}

A total of twenty-seven samples of industrial C. sativa female inflorescences of different varieties, including Kompolti $(n=9)$, Tiborszallasi $(n=7)$, Antal $(n=7)$, and Carmagnola Cs $(n=4)$ were provided by several hemp farmers located in Italy. All inflorescences were harvested in October 2019 and fulfilled the requirements set at EC regulation (No 809/2014) [48] regarding their psychoactive cannabinoids content. The samples were dried at $36{ }^{\circ} \mathrm{C}$ using a forced-air laboratory oven until the sample moisture reached a level from $8 \%$ to $12 \%$. The samples were milled into powder using a laboratory mill (particle size $200 \mu \mathrm{m}$ ) and then stored at $4{ }^{\circ} \mathrm{C}$ until analysis. 
The standards of polyphenols (purity $>98 \%$ ) were purchased from Sigma Aldrich (Milan, Italy), and included: chlorogenic acid, caffeic acid, $p$-coumaric acid, ferulic acid (Hydroxycinnamic Acids); rutin, quercetin-3-glucoside, kaempferol-3-O-glucoside, quercetin, kaempferol (Flavonol); luteolin-7-O-glucoside, apigenin-7-O-glucoside, luteolin, apigenin (Flavones); catechin, epicatechin (Flavanols) and naringenin (Flavanone).

Due to the lack of analytical standards, the identification of polyphenols $(n=6)$ including cannabisin A, cannabisin B, cannabisin C (Lignanamide), cannflavin A, cannflavin B (Flavanone) and $\mathrm{N}$-trans-caffeoyltyramine (Phenolic amides) was carried out by a post-target screening.

Methanol (MeOH), water (LC-MS grade) were acquired from Merk (Darmstadt, Germany), and formic acid (mass spectrometry grade) was purchased from Fluka (Milan, Italy).

\subsection{Polyphenols Extraction}

Extraction has been carried out according to the procedure described by Calzolari et al., [49] with some modifications. Briefly, $100 \mathrm{mg}$ of sample was suspended in $15 \mathrm{~mL}$ of methanol, the mixture was vortexed intensively for $3 \mathrm{~min}$ and sonicated in the dark, at $4{ }^{\circ} \mathrm{C}$, for $30 \mathrm{~min}$. Then, the mixture was centrifuged at $5000 \times \mathrm{g}$ at $4{ }^{\circ} \mathrm{C}$ for $10 \mathrm{~min}$. The supernatants were pooled, filtered through $0.2 \mu \mathrm{m}$ syringe filters ( $26 \mathrm{~mm}, \mathrm{RC}$ membrane, Phenomenex, Castel Maggiore, Italy), and an aliquot introduced into a chromatography vial.

\subsection{Determination of Total Phenolic Content (TPC)}

Total phenolic content was performed according to the Folin-Ciocalteu method [50] with slight modifications. Briefly, $125 \mu \mathrm{L}$ of extract sample was diluted in $500 \mu \mathrm{L}$ of deionized water, then $125 \mu \mathrm{L}$ of the Folin-Ciocalteu reagent was added to the mixture, followed by $6 \mathrm{~min}$ of incubation at room temperature. Afterward, $1.25 \mathrm{~mL}$ of $7.5 \%$ of sodium carbonate solution and $1 \mathrm{~mL}$ of deionized water were added in the mixture. The absorbance at $760 \mathrm{~nm}$ after $90 \mathrm{~min}$ of incubation in the dark was measured. The TPC of inflorescence samples was expressed as mg of gallic acid equivalents (GAE)/g of sample.

\subsection{Determination of 1,1-Diphenyl-2-picrylhydrazyl (DPPH) Free Radical-Scavenging}

The radical-scavenging activity of the sample extract was determined using the method suggested by Brand-Williams et al. [51] with some modifications. Briefly, to obtain the DPPH radical working solution, the DPPH standard (4 mg in $10 \mathrm{~mL}$ ) was diluted with methanol until the absorbance value reached $0.90( \pm 0.02)$ at $517 \mathrm{~nm}$. Then, $200 \mu \mathrm{L}$ of sample extract was added to $1 \mathrm{~mL}$ of DPPH radical working solution. The mixture was shaken vigorously, and then the decrease absorbance after $10 \mathrm{~min}$ at $517 \mathrm{~nm}$ was measured. The results were expressed as mmol trolox equivalents (TE)/ $\mathrm{kg}$ of the sample.

\subsection{Ultra-High Performance Liquid Chromatography and Orbitrap High-Resolution Mass Spectrometry Analysis}

The polyphenolic profile was analyzed by Ultra High-Pressure Liquid Chromatograph (UHPLC, Dionex UltiMate 3000, Thermo Fisher Scientific, Waltham, MA, USA) equipped with a degassing system, a Quaternary UHPLC pump working at $1250 \mathrm{bar}$, and an autosampler device. Chromatographic separation of polyphenols was performed with a thermostated $\left(\mathrm{T}=25^{\circ} \mathrm{C}\right)$ Kinetex $2.6 \mu \mathrm{m}$ Biphenyl $(100 \times 2.1 \mathrm{~mm}$, Phenomenex) column. The injection volume was $2 \mu \mathrm{L}$. The mobile phase consisted of a binary solution: water (phase A) and methanol (phase B), both mobile phases contained $0.1 \%$ of formic acid. A gradient elution program was applied as follows: an initial 5\% B, increased to 30\% B in $1.3 \mathrm{~min}$, and a new to $100 \% \mathrm{~B}$ in $8 \mathrm{~min}$. The gradient was held for $2 \mathrm{~min}$ at $100 \% \mathrm{~B}$ and reduced to $5 \% \mathrm{~B}$ in $2 \mathrm{~min}$. The flow rate of $0.2 \mathrm{~mL} / \mathrm{min}$. Afterward, the gradient switched back to $5 \%$ in $2 \mathrm{~min}$, and another $2 \mathrm{~min}$ for column re-equilibration at $5 \%$. The UHPLC system was coupled to a Q-Exactive Orbitrap mass spectrometer (UHPLC, Thermo Fischer Scientific, Waltham, MA, USA). An ESI source (Thermo Fisher Scientific, Waltham, MA, USA) was operated in negative ion mode (ESI-) setting two 
scan events (Full ion MS and All ion fragmentation, AIF) for all compounds of interest. Full scan data were acquired at a resolving power of 35,000 FWHM (full width at half maximum). Ion source parameters were spray voltage $2.8 \mathrm{kV}$ (negative mode), capillary temperature $310{ }^{\circ} \mathrm{C}$, S-lens RF level 50 , sheath gas pressure $\left(\mathrm{N}_{2}>95 \%\right) 35$, auxiliary gas $\left(\mathrm{N}_{2}>95 \%\right) 10$, auxiliary gas heater temperature $350{ }^{\circ} \mathrm{C}$. The value for the automatic gain control (AGC) target was set at $3 \times 10^{6}$, a scan range of $\mathrm{m} / \mathrm{z} 90$ to 1000 was chosen, and the injection time was set to $200 \mathrm{~ms}$. The scan-rate was set at $2 \mathrm{scans} / \mathrm{s}$. Data analysis and processing were performed using Xcalibur software, v. 3.1.66.10.

For the scan event of AIF, the parameters were set as follows: mass resolving power of 17,500 FWHM at $200 \mathrm{~ms}$; scan time $=0.10 \mathrm{~s}$. The collision energy was varied in the range of 10 to $45 \mathrm{eV}$ to obtain representative product ion spectra. Data processing was performed by the Quan/Qual Browser Xcalibur software, v. 3.1.66.10 (Xcalibur, Thermo Fisher Scientific, Waltham, MA, USA). Detection was based on calculated exact mass with a mass error below $5 \mathrm{ppm}$ and on the retention time of the molecular ion; while regarding the fragments on the intensity threshold of 1000 and a mass tolerance of $5 \mathrm{ppm}$. Quantitative results were obtained working in scan mode with HRMS exploiting the high selectivity achieved in full-scan mode, whereas MS/HRMS information was used for confirmatory purposes.

\subsection{Statistics and Data Analysis}

Values were expressed as the average values and concentration range of triplicate measurements. The differences between average values were evaluated by using Tukey's test at the level of significance $p<0.05$. Statistical analysis was performed using STATA 12 (STATA corp LP, College Station, TX, USA).

\section{Conclusions}

Even if studies regarding the beneficial effects of hemp seeds, oils, and leaves are numerous, and research on C. sativa extract is constantly in progress, there are few references concerning the biological activities and the potential health benefits of $C$. sativa inflorescence. A comprehensive analysis of the bioactivity for Kompolti, Tiborszallasi, Antal, and Carmagnola Cs cultivar of C. sativa inflorescences and polyphenols characterization through UHPLC-Q-Orbitrap spectrometry measurement were carried out in this research work for the first time.

A comparison of the studied cultivars showed that Carmagnola CS had the highest investigated polyphenols amount (sum average of $743.5 \mathrm{mg} / \mathrm{kg}$ ), TPC content ( $33.2 \pm 0.5 \mathrm{mg} \mathrm{GAE} / \mathrm{g}$ ) as well as free radical-scavenging activity $(63.6 \pm 0.9 \mathrm{mmol}$ trolox $/ \mathrm{kg})$, thus, appeared to be the most promising cultivar. In spite of the renovated interest for this cultivar, data about the correlation on bioactivity and cultivar are still fragmentary.

Our results highlighted the possibility of also using this part of the plant, which represents a valuable source of natural antioxidants and a rich fount of polyphenols, including cannflavins, which represent bioactive compounds not common in other typical plants. It is, therefore, desirable to continue to expand the understanding of this actual topic to estimate their efficacy for future applications for nutraceutical purposes.

Supplementary Materials: The supplementary materials are available online.

Author Contributions: A.R. and Y.R.C. conceived and designed the experiments; L.I. performed the experiments; G.G. and A.G. analyzed the data, L.I., L.C., A.N. wrote the paper and Y.R.C. review and editing. All authors have read and agreed to the published version of the manuscript.

Funding: This research received no external funding.

Conflicts of Interest: The authors declare no conflict of interest. 


\section{References}

1. Andre, C.M.; Hausman, J.-F.; Guerriero, G. Cannabis sativa: The Plant of the Thousand and One Molecules. Front Plant Sci 2016, 7, 19. [CrossRef] [PubMed]

2. Cushnie, T.T.; Lamb, A.J. Antimicrobial activity of flavonoids. Int. J. Antimicrob. Agents 2005, 26, $343-356$. [CrossRef] [PubMed]

3. Mkpenie, V.; Essien, E.; Udoh, I. Effect of extraction conditions on total polyphenol contents, antioxidant and antimicrobial activities of Cannabis sativa L. Electron. J. Environ. Agric. Food Chem. 2012, 11, 300.

4. Siano, F.; Moccia, S.; Picariello, G.; Russo, G.L.; Sorrentino, G.; Di Stasio, M.; La Cara, F.; Volpe, M.G. Comparative Study of Chemical, Biochemical Characteristic and ATR-FTIR Analysis of Seeds, Oil and Flour of the Edible Fedora Cultivar Hemp (Cannabis sativa L.). Molecules 2018, 24, 83. [CrossRef]

5. Pollastro, F.; Minassi, A.; Fresu, L.G. Cannabis phenolics and their bioactivities. Curr. Med. Chem. 2018, 25, 1160-1185. [CrossRef]

6. Mandal, S.M.; Chakraborty, D.; Dey, S. Phenolic acids act as signaling molecules in plant-microbe symbioses. Plant Signal. Behav. 2010, 5, 359-368. [CrossRef]

7. Castaldo, L.; Narváez, A.; Izzo, L.; Graziani, G.; Gaspari, A.; Minno, G.D.; Ritieni, A. Red Wine Consumption and Cardiovascular Health. Molecules 2019, 24, 3626. [CrossRef]

8. Santini, A.; Tenore, G.C.; Novellino, E. Nutraceuticals: A paradigm of proactive medicine. Eur. J. Pharm. Sci. 2017, 96, 53-61. [CrossRef]

9. Kelly, N.P.; Kelly, A.L.; O’Mahony, J.A. Strategies for enrichment and purification of polyphenols from fruit-based materials. Trends Food Sci. Technol. 2019, 83, 248-258. [CrossRef]

10. Lin, J.; Teo, L.M.; Leong, L.P.; Zhou, W. In vitro bioaccessibility and bioavailability of quercetin from the quercetin-fortified bread products with reduced glycemic potential. Food Chem. 2019, 286, 629-635. [CrossRef]

11. Gómez-Juaristi, M.; Martínez-López, S.; Sarria, B.; Bravo, L.; Mateos, R. Bioavailability of hydroxycinnamates in an instant green/roasted coffee blend in humans. Identification of novel colonic metabolites. Food Funct. 2018, 9, 331-343. [CrossRef]

12. Shukla, M.; Jaiswal, S.; Sharma, A.; Srivastava, P.K.; Arya, A.; Dwivedi, A.K.; Lal, J. A combination of complexation and self-nanoemulsifying drug delivery system for enhancing oral bioavailability and anticancer efficacy of curcumin. Drug Dev. Ind. Pharm. 2017, 43, 847-861. [CrossRef]

13. Abbas, M.; Saeed, F.; Anjum, F.M.; Afzaal, M.; Tufail, T.; Bashir, M.S.; Ishtiaq, A.; Hussain, S.; Suleria, H.A.R. Natural polyphenols: An overview. Int. J. Food Prop. 2017, 20, 1689-1699. [CrossRef]

14. Tenore, G.C.; Campiglia, P.; Ciampaglia, R.; Izzo, L.; Novellino, E. Antioxidant and antimicrobial properties of traditional green and purple "Napoletano" basil cultivars (Ocimum basilicum L.) from Campania region (Italy). Nat. Prod. Res. 2017, 31, 2067-2071. [CrossRef]

15. Castaldo, L.; Graziani, G.; Gaspari, A.; Izzo, L.; Luz, C.; Mañes, J.; Rubino, M.; Meca, G.; Ritieni, A. Study of the Chemical Components, Bioactivity and Antifungal Properties of the Coffee Husk. J. Food Res. 2018, 7, 43-54. [CrossRef]

16. Fathordoobady, F.; Singh, A.; Kitts, D.D.; Pratap Singh, A. Hemp (Cannabis sativa L.) Extract: Anti-Microbial Properties, Methods of Extraction, and Potential Oral Delivery. Food Rev. Int. 2019, 35, 664-684. [CrossRef]

17. Frassinetti, S.; Moccia, E.; Caltavuturo, L.; Gabriele, M.; Longo, V.; Bellani, L.; Giorgi, G.; Giorgetti, L. Nutraceutical potential of hemp (Cannabis sativa L.) seeds and sprouts. Food Chem. 2018, 262, 56-66. [CrossRef]

18. Nagy, D.U.; Cianfaglione, K.; Maggi, F.; Sut, S.; Dall'Acqua, S. Chemical Characterization of Leaves, Male and Female Flowers from Spontaneous Cannabis (Cannabis sativa L.) Growing in Hungary. Chem. Biodivers. 2019, 16, e1800562. [CrossRef]

19. Piccolella, S.; Crescente, G.; Candela, L.; Pacifico, S. Nutraceutical polyphenols: New analytical challenges and opportunities. J. Pharm. Biomed. Anal. 2019, 175, 112774. [CrossRef]

20. López-Gutiérrez, N.; Romero-González, R.; Plaza-Bolaños, P.; Martínez Vidal, J.L.; Garrido Frenich, A. Identification and quantification of phytochemicals in nutraceutical products from green tea by UHPLC-Orbitrap-MS. Food Chem. 2015, 173, 607-618. [CrossRef]

21. López-Gutiérrez, N.; Romero-González, R.; Martínez Vidal, J.L.; Frenich, A.G. Determination of polyphenols in grape-based nutraceutical products using high resolution mass spectrometry. LWT Food Sci. Technol. 2016, 71, 249-259. [CrossRef] 
22. Stanojević, M.; Trifkovic, J.; Dramićanin, A.; Gašić, U.; Fotiric Aksic, M.; Milojković-Opsenica, D. Determination of the phenolic profile of peach (Prunus persica L.) kernels using UHPLC-LTQ OrbiTrap MS/MS technique. Eur. Food Res. Technol. 2018, 244, 2051-2064.

23. Rodríguez-Carrasco, Y.; Gaspari, A.; Graziani, G.; Santini, A.; Ritieni, A. Fast analysis of polyphenols and alkaloids in cocoa-based products by ultra-high performance liquid chromatography and Orbitrap high resolution mass spectrometry (UHPLC-Q-Orbitrap-MS/MS). Food Res. Int. 2018, 111, 229-236. [CrossRef] [PubMed]

24. Flores-Sanchez, I.J.; Verpoorte, R. Secondary metabolism in cannabis. Phytochem. Rev. 2008, 7, 615-639. [CrossRef]

25. Irakli, M.; Tsaliki, E.; Kalivas, A.; Kleisiaris, F.; Sarrou, E.; Cook, C.M. Effect of Genotype and Growing Year on the Nutritional, Phytochemical, and Antioxidant Properties of Industrial Hemp (Cannabis sativa L.) Seeds. Antioxidants 2019, 8, 491. [CrossRef]

26. Yan, X.; Tang, J.; dos Santos Passos, C.; Nurisso, A.; Simoes-Pires, C.A.; Ji, M.; Lou, H.; Fan, P. Characterization of lignanamides from hemp (Cannabis sativa L.) seed and their antioxidant and acetylcholinesterase inhibitory activities. J. Agric. Food Chem. 2015, 63, 10611-10619. [CrossRef] [PubMed]

27. Chen, H.; Li, Y.-J.; Sun, Y.-J.; Gong, J.-H.; Du, K.; Zhang, Y.-L.; Su, C.-F.; Han, Q.-Q.; Zheng, X.-K.; Feng, W.-S. Lignanamides with potent antihyperlipidemic activities from the root bark of Lycium chinense. Fitoterapia 2017, 122, 119-125. [CrossRef]

28. Zhang, J.-X.; Guan, S.-H.; Feng, R.-H.; Wang, Y.; Wu, Z.-Y.; Zhang, Y.-B.; Chen, X.-H.; Bi, K.-S.; Guo, D.-A. Neolignanamides, lignanamides, and other phenolic compounds from the root bark of Lycium chinense. J. Nat. Prod. 2013, 76, 51-58. [CrossRef] [PubMed]

29. Sun, J.; Gu, Y.-F.; Su, X.-Q.; Li, M.-M.; Huo, H.-X.; Zhang, J.; Zeng, K.-W.; Zhang, Q.; Zhao, Y.-F.; Li, J. Anti-inflammatory lignanamides from the roots of Solanum melongena L. Fitoterapia 2014, 98, 110-116. [CrossRef] [PubMed]

30. Gao, K.; Ma, D.; Cheng, Y.; Tian, X.; Lu, Y.; Du, X.; Tang, H.; Chen, J. Three new dimers and two monomers of phenolic amides from the fruits of Lycium barbarum and their antioxidant activities. J. Agric. Food Chem. 2015, 63, 1067-1075. [CrossRef] [PubMed]

31. Biesiada, A.; Tomczak, A. Biotic and abiotic factors affecting the content of the chosen antioxidant compounds in vegetables. Veg. Crop. Res. Bull. 2012, 76, 55-78. [CrossRef]

32. Rea, K.A.; Casaretto, J.A.; Al-Abdul-Wahid, M.S.; Sukumaran, A.; Geddes-McAlister, J.; Rothstein, S.J.; Akhtar, T.A. Biosynthesis of cannflavins A and B from Cannabis sativa L. Phytochemistry 2019, 164, 162-171. [CrossRef]

33. Werz, O.; Seegers, J.; Schaible, A.M.; Weinigel, C.; Barz, D.; Koeberle, A.; Allegrone, G.; Pollastro, F.; Zampieri, L.; Grassi, G. Cannflavins from hemp sprouts, a novel cannabinoid-free hemp food product, target microsomal prostaglandin E2 synthase-1 and 5-lipoxygenase. PharmaNutrition 2014, 2, 53-60. [CrossRef]

34. Barrett, M.; Gordon, D.; Evans, F. Isolation from Cannabis sativa L. of cannflavin-a novel inhibitor of prostaglandin production. Biochem. Pharmacol. 1985, 34, 2019-2024. [CrossRef]

35. Barrett, M.; Scutt, A.; Evans, F. Cannflavin A and B, prenylated flavones from Cannabis sativa L. Experientia 1986, 42, 452-453. [CrossRef] [PubMed]

36. Eggers, C.; Fujitani, M.; Kato, R.; Smid, S. Novel cannabis flavonoid, cannflavin A displays both a hormetic and neuroprotective profile against amyloid $\beta$-mediated neurotoxicity in PC12 cells: Comparison with geranylated flavonoids, mimulone and diplacone. Biochem. Pharmacol. 2019, 169, 113609. [CrossRef]

37. Vonapartis, E.; Aubin, M.-P.; Seguin, P.; Mustafa, A.F.; Charron, J.-B. Seed composition of ten industrial hemp cultivars approved for production in Canada. J. Food Compos. Anal. 2015, 39, 8-12. [CrossRef]

38. Gonçalves, J.; Rosado, T.; Soares, S.; Simão, A.Y.; Caramelo, D.; Luís, Â.; Fernández, N.; Barroso, M.; Gallardo, E.; Duarte, A.P. Cannabis and its secondary metabolites: Their use as therapeutic drugs, toxicological aspects, and analytical determination. Medicines 2019, 6, 31. [CrossRef]

39. Moccia, S.; Siano, F.; Russo, G.L.; Volpe, M.G.; La Cara, F.; Pacifico, S.; Piccolella, S.; Picariello, G. Antiproliferative and antioxidant effect of polar hemp extracts (Cannabis sativa L. Fedora cv.) in human colorectal cell lines. Int. J. Food Sci. Nutr. 2019, 1-14. [CrossRef]

40. Smeriglio, A.; Galati, E.M.; Monforte, M.T.; Lanuzza, F.; D’Angelo, V.; Circosta, C. Polyphenolic Compounds and Antioxidant Activity of Cold-Pressed Seed Oil from Finola Cultivar of Cannabis sativa L. Phytother. Res. 2016, 30, 1298-1307. [CrossRef] 
41. Drinić, Z.; Vidović, S.; Vladić, J.; Koren, A.; Kiprovski, B.; Sikora, V. Effect of extraction solvent on total polyphenols content and antioxidant activity of Cannabis sativa L. Lek. Sirovine 2018, 38, 17-21. [CrossRef]

42. Ferrante, C.; Recinella, L.; Ronci, M.; Menghini, L.; Brunetti, L.; Chiavaroli, A.; Leone, S.; Di Iorio, L.; Carradori, S.; Tirillini, B. Multiple pharmacognostic characterization on hemp commercial cultivars: Focus on inflorescence water extract activity. Food Chem. Toxicol. 2019, 125, 452-461. [CrossRef] [PubMed]

43. Mikulec, A.; Kowalski, S.; Sabat, R.; Skoczylas, Ł.; Tabaszewska, M.; Wywrocka-Gurgul, A. Hemp flour as a valuable component for enriching physicochemical and antioxidant properties of wheat bread. Lwt-Food Sci. Technol. 2019, 102, 164-172. [CrossRef]

44. De Beer, D.; Harbertson, J.F.; Kilmartin, P.A.; Roginsky, V.; Barsukova, T.; Adams, D.O.; Waterhouse, A.L. Phenolics: A comparison of diverse analytical methods. Am. J. Enol. Vitic. 2004, 55, 389-400.

45. Olszowy, M. What is responsible for antioxidant properties of polyphenolic compounds from plants? Plant Physiol. Biochem. 2019, 144, 135-143. [CrossRef]

46. Heleno, S.A.; Martins, A.; Queiroz, M.J.R.; Ferreira, I.C. Bioactivity of phenolic acids: Metabolites versus parent compounds: A review. Food Chem. 2015, 173, 501-513. [CrossRef]

47. Burri, S.C.; Ekholm, A.; Håkansson, Å.; Tornberg, E.; Rumpunen, K. Antioxidant capacity and major phenol compounds of horticultural plant materials not usually used. J. Funct. Foods 2017, 38, 119-127. [CrossRef]

48. European Commission (EC). European Commission (EC) No. 809/2014. Offic. J. Eur. Union 2014, L227, 69-124.

49. Calzolari, D.; Magagnini, G.; Lucini, L.; Grassi, G.; Appendino, G.; Amaducci, S. High added-value compounds from Cannabis threshing residues. Ind. Crop. Prod. 2017, 108, 558-563. [CrossRef]

50. Singleton, V.L.; Rossi, J.A. Colorimetry of total phenolics with phosphomolybdic-phosphotungstic acid reagents. Am. J. Enol. Vitic. 1965, 16, 144-158.

51. Brand-Williams, W.; Cuvelier, M.-E.; Berset, C. Use of a free radical method to evaluate antioxidant activity. LWT Food Sci. Technol. 1995, 28, 25-30. [CrossRef]

Sample Availability: Not available.

(C) 2020 by the authors. Licensee MDPI, Basel, Switzerland. This article is an open access article distributed under the terms and conditions of the Creative Commons Attribution (CC BY) license (http://creativecommons.org/licenses/by/4.0/). 